Pacific Journal of Mathematics

ATTAINING THE SPREAD AT CARDINALS WHICH ARE NOT 


\title{
ATTAINING THE SPREAD AT CARDINALS WHICH ARE NOT STRONG LIMITS
}

\author{
JUDITH ROITMAN
}

It is shown to be consistent with set theory that there is a cardinal $\kappa$ and a Hausdorff space $X$ such that $\mathrm{ef}(\kappa)>\omega$ and $\operatorname{sp}(X)=\kappa$ and $X$ contains no discrete subspace of cardinality $\kappa$; also, if $X$ is a Hausdorff space such that $\operatorname{cf}(\operatorname{sp}(X)=$ $\omega$ and $X$ does not attain its spread, then $X$ contains a subspace of a certain canonical form with the same spread.

1. Preliminaries. The spread of a topological space $X$, abbreviated as $\operatorname{sp}(X)$ is defined as a supremum of cardinalities of certain subspaces:

$$
\operatorname{sp}(X)=\sup \{|Y|: Y \text { is a discrete subspace of } X\}
$$

where $|Y|$ is the cardinality of $Y$. For brevity, we say that spread must be attained at $\kappa$ iff every Hausdorff space $X$ for which $\mathrm{sp}(X)=\kappa$ has a discrete subspace of cardinality $\kappa$. A natural question to ask, then, is whether spread must be attained at every cardinal $\kappa$. The answer is clearly yes if $\kappa$ is a successor cardinal. If $\kappa$ is a limit cardinal, it is trivial to construct spaces which are, say, $T_{1}$ but not $T_{2}$ which have a spread of $\kappa$ but no discrete subspace of cardinality $\kappa$, thus necessitating the word "Hausdorff" in our definition of attaining the spread. Juhasz and Hajnal have found classes of limit cardinals at which spread is attained; they also have a class of spaces for which, if the spread has cofinality $\omega$, then the space has a discrete subspace of the cardinality of the spread (we say that the space attains its spread). Here we look for counterexamples: it is found consistent with the axioms of set theory to have Hausdorff spaces of uncountable cofinality which do not attain their spread; in the case of countable cofinality, it is shown that a Hausdorff space which does not attain its spread contains a space of a certain canonical form which has the same spread.

Notation and conventions. Lower case Greek letters are reserved for ordinals, which may or may not be cardinals; $\kappa$ is reserved for cardinals, which are assumed to be initial ordinals.

We remind the reader of some basic concepts about ordinals.

Definition 1. $\operatorname{cf}(\alpha)=\beta$ iff $\beta$ is the least ordinal such that 
for some function $f: \beta \rightarrow \alpha, f$ is increasing and sup (range $f$ ) $=\alpha$. cf $(\alpha)$ is the cofinality of $\alpha$.

Definition 2. $\alpha$ is regular iff $\alpha=\operatorname{cf}(\alpha) . \quad \alpha$ is singular otherwise. We note that regular ordinals are always cardinals.

DEFINITION 3. $\kappa$ is a limit cardinal iff for every cardinal $\tau, \kappa \neq \tau^{+} . \quad \kappa$ is a strong limit cardinal iff for every cardinal $\tau<\kappa, 2^{\tau}<\kappa$.

DEFINITION 4. $\kappa$ is weakly inaccessible iff $\kappa$ is a regular limit cardinal. $\kappa$ is strongly inaccessible iff $\kappa$ is a regular strong limit cardinal.

Since our purpose is to find counterexamples or describe what they must look like if we could find them, it would be useful to know where not to look. The following result of Hajnal and Juhasz tells us, and also insures that a counterexample must be a consistency result, i.e. it cannot exist in all models of set theory.

Theorem 5. (Juhasz, Hajnal [2 and 3]) If $\kappa$ is a weakly compact or a singular strong limit cardinal, and $X$ is a Hausdorff space of cardinality $\kappa$, then $X$ has a discrete subspace of cardinality $\kappa$.

Thus in model of GCH spread is attained at singular cardinals. In fact in the constructible universe $L$ all our questions about spread are settled, since a cardinal $\kappa$ of $L$ which is a regular limit cardinal and not weakly compact has a $\kappa$-Suslin line, and this line has spread $\kappa$ which is not attained (see Juhasz [6]). Weakly compact cardinals play no further role in this paper, and the curious reader is referred to Juhasz [6] for a definition.

The results in this paper were originally proved in longer proofs using combinatorics in an inelegant fashion. The author is grateful to Ken Kunen and Istvan Juhasz for pointing out how they could be shortened; she also thanks the referee for helpful comments on organization.

2. The case ef $(\kappa)>\omega$. Theorem 5 tells us that in order to not attain the spread at $\kappa, \mathrm{GCH}$ must be violated below $\kappa$ in a strong fashion. Theorem 6 will set up machinery for constructing spaces which do not attain their spread from spaces of small spread and large cardinality. Corollary 7 will show that there is a class of cardinals for which this construction works which is large in the sense that Easton forcing makes it easy for us to find models of 
set theory in which this class is cofinal in the class of all ordinals. Corollary 8 will point to a subclass of the class of Corollary 7 whose consistency follows from any large cardinal axiom. Corollary 9 will connect the machinery to the existence of large spaces with small width, which has been shown to be consistent by Hajnal and Juhasz [5].

THEOREM 6. Let $\kappa$ be a limit cardinal of uncountable cofinality, and suppose there exists a Hausdorff space $\langle X, \mathscr{T}\rangle,|X|=\kappa$, such that the spread of $\langle X, \mathscr{T}\rangle$ is less than the cofinality of $\kappa$. Then there is a finer topology $\mathscr{T}^{\prime}$ on $X$ such that $\left\langle X, \mathscr{T}^{\prime}\right\rangle$ has a spread of $\kappa$ which is not attained.

Proof. Let $\xi=\mathrm{cf}(\kappa)$. We may write the set $X$ then as the disjoint sum $X=\sum_{\alpha<\xi} X_{\alpha}$, where $\left|X_{\alpha}\right|=\kappa_{\alpha}$ for every $\alpha<\xi$, $\kappa=$ $\sup \left\{\kappa_{\alpha}: \alpha<\xi\right\}$, and if $\alpha<\beta<\xi$ then $\kappa_{\alpha}<\kappa_{\beta}$. Let $\mathscr{T}^{\prime}$ be the topology on $X$ derived from sub-basic sets of the following form:

Suppose $x \in X$. Then for some unique $\alpha, x \in X_{\alpha}$.

Let $u \in \mathscr{T}, x \in u$. Then $\{x\} \cup\left(u-X_{\alpha}\right) \in \mathscr{T}^{\prime}$. Since each $\left\langle W_{\alpha}\right.$, $\left.\mathscr{T}^{\prime}\right\rangle$ is discrete, $\left\langle X, \mathscr{T}^{\prime}\right\rangle$ has spread $\kappa .\left\langle X, \mathscr{T}^{\prime}\right\rangle$ is Hausdorff because $\langle X, \mathscr{T}\rangle$ is. We need to show that $\left\langle X, \mathscr{T}^{\prime}\right\rangle$ does not attain its spread.

Proceeding by contradiction, suppose $Y$ is a discrete subspace of $\left\langle X, \mathscr{T}^{\prime}\right\rangle$ of cardinality $\kappa$. Then since $\mathrm{cf}(\kappa)=\xi$, there is some $Y^{\prime} \subset Y$ with $\left|Y^{\prime}\right|=\xi$ and for every $\alpha<\xi,\left|Y^{\prime} \cap X_{\alpha}\right| \leqq 1$. If $Y$ is discrete, so is $Y^{\prime}$. Let $U=\left\{u_{x}: x \in Y^{\prime}\right\}$ be a subset of $\mathscr{T}^{\prime}$ where $x \in u_{x}$ and if $y \neq x$ then $u_{x} \cap u_{y} \cap Y^{\prime}=\varnothing$. We may assume each $u_{x}$ is a basic open set, hence of the form

$$
u_{x}=Z_{x} \cup\left(u_{x}^{*}-\bigcup_{\alpha \in A_{x}} X_{\alpha}\right)
$$

where $A_{x}$ is a finite subset of $\xi, Z_{x}$ a finite subset of $\bigcup_{\alpha \in A_{x}} X_{\alpha}$, $x \in Z_{x} \cap u_{x}^{*}$, and $u_{x}^{*} \in \mathscr{T}$. Hence $Y^{\prime} \subset u_{x}^{*} \cap Z_{x}$ for every $x \in Y^{\prime}$

But then each $u_{x}^{*} \cap Y^{\prime}$ is finite, and since $Y^{\prime}$ has a cover by sets in $\mathscr{T}$ which are finite when relativized to $Y^{\prime}$, it is easily seen that $Y^{\prime}$ is a discrete subspace of $\langle X, \mathscr{T}\rangle$ of cardinality, $\xi$. But this contradicts the hypothesis that $\xi\rangle$ sp $\langle X, \mathscr{T}\rangle$ and the proof of Theorem 6 is complete.

COROLlaRY 7. Let $\kappa$ be a limit cardinal of uncountable cofinality, and suppose there is some $\tau<\operatorname{cf}(\kappa)$ such that $2^{\tau}>\kappa$. Then spread 
is not attained at $\kappa$.

Proof. The set of functions $2^{\tau}$ under the product topology has a basis of cardinality $\tau$, since the set of functions into 2 whose domains are finite subsets of $\tau$ is isomorphic to a basis. But the spread of a space cannot be larger than the cardinality of some basis, so any subspace of $2^{\tau}$ has spread $\leqq \tau$. In particular, any $X \subset 2^{\tau}$ where $|X|=\kappa$ can be used in the hypothesis of Theorem 6 .

Now Easton forcing (1) gives us a technique for the following: let $M$ be a transitive model of $\mathrm{ZFC}+\mathrm{GCH}$ and let $F$ be a nondecreasing function whose domain is the cardinals of $M$ such that if $\kappa$ is a regular cardinal of $M$ then $\operatorname{cf}(F(\kappa))>\kappa$. Then there is a model of set theory, $N$, which has the same cardinals as $M$, where cardinals have the same cofinalities they had in $M$, and in which if $\kappa$ is regular they $2^{\kappa}=F(\kappa)$. Using this technique it is easy to get models of set theory in which the class of cardinals satisfying Corollary 7 is cofinal in the class of cardinals of the model.

COROLLARY 8. Let $\kappa$ be weakly inaccessible but not strongly inaccessible. Then the spread need not be attained at $\kappa$.

Proof. Then the hypothesis of Corollary 7 is satisfied.

For a last example of cardinals to which Theorem 6 applies, we look at a model of set theory due to Juhasz and Hajnal and found in [5]. Here a forcing argument is used over a model $M$ in which $2^{\kappa}=\kappa^{+}$to make a model with the same cardinals and cofinalities in which $2^{\kappa}$ is still $\kappa^{+}$but $2^{\left(\kappa^{+}\right)}$is now "as large as you want" and there is a hereditarily $\kappa$-separable Hausdorff space (equivalently a space of width $\kappa$ ) of cardinality $2^{\left(\kappa^{+}\right)}$. In particular, we may make $2^{\left(\kappa^{+}\right)}>\omega_{\kappa^{+}}$. Since a width of $\kappa$ implies a spread which is $\leqq \kappa$, this model justifies the conclusion of

COROLLARY 9. It is consistent with the axioms of set theory to have a cardinal $\kappa$ such that $2^{\kappa}=\kappa^{+}$and spread is not attained at $\omega_{\kappa^{+}}$.

An explicit examination, not performed here, of each of the spaces of Corollaries 7 and 9 shows that none of them is regular. It will also be noted that in these corollaries a cardinal bounded by the cofinality of $\kappa$ has the large power set necessary to avoid Theorem 5. So the following open questions remain:

Must spread be attained in the class of regular spaces? 
What happens when $\kappa$ is not a strong limit but $2^{\tau}<\kappa$ for all $\tau<\operatorname{cf}(\kappa) ?$

3. The case ef $(\kappa)=\omega$. Here we do not have a counterexample, but if one exists we know what it must look like.

THEOREM 10. Suppose $X$ is a Hausdorff space whose spread has cofinality $\omega$. Then $X$ contains a downward subspace with the same spread.

Theorem 10 tells us that if we want a counterexample to attaining a spread of countable cofinality we need only look at the class of downward spaces, which we define forthwith.

Definition 11. Let $X$ be a topological space. Then $X$ is downward iff $X$ is set-theoretically the disjoint sum $\sum_{n \in \omega} X_{n}$ where each $X_{n}$ is a discrete subspace and for every $m \in \omega, \mathrm{U}_{n<m} X_{n}$ is open.

The proof of Theorem 10 relies on a combinatorial theorem of Hajnal, which we state as

THEOREM 12. (Hajnal) Let $f$ be a function mapping a set $X$ into its power set and such that for some cardinal $\tau<|X|$, $|f(x)|<\tau$ for every $x \in X$. Then there is a set $Y \leqq X,|Y|=|X|$, such that if $x, y$ are elements of $Y$ then $x \notin f(y)$. Then set $Y$ is called a free set for $f$.

It is clear that if $f$ is a function taking each element of $X$ into an open neighborhood, then the set $Y$ which is free for $f$ is also discrete in the topology for $X$. The proof of Theorem 12 can be found in Juhasz [6].

Proof of Theorem 10. Let $X$ be a Hausdorff space whose spread has cofinality $\omega, \operatorname{sp}(X)=\kappa$, and suppose $\kappa$ is the limit of the strictly increasing sequence $\kappa_{n}, n \in \omega$. Then $X$ contains disjoint discrete subspaces $X_{n}$ where each $X_{n}$ has cardinality $\kappa_{n}$, so without loss of generality we assume $X$ is the union of these $X_{n}$ 's. There are three cases to consider.

Case 1. Every open set in $X$ has cardinality $\kappa$. Then since $X$ is Hausdorff it has a countable infinite family of disjoint open sets, call them $u_{n}, n \in \omega$. Then each $u_{n}$, having cardinality $\kappa$, contains at least $\kappa_{n}$ elements from some $X_{m_{n}}$, say $u_{n} \cap X_{m_{n}}=Y_{n}$. Then 
$Y=\bigcup_{n \in \omega} Y_{n}$ is discrete, and hence trivially downward.

Case 2. For some $\tau<\kappa$, every point in $X$ has a neighborhood of cardinality strictly less than $\tau$. Then we let $f$ be the map taking each point in $X$ into such a small neighborhood. By Theorem 12 we then have a free set $Y$ for $f$ of cardinality $\kappa$, which as we have noted is a discrete subspace and hence downward.

Case 3. For every $n<\omega \mid\{x \in X: x$ has a neighborhood of cardinality $\left.\geqq \kappa_{n}\right\} \mid=\kappa$. By Case 1 we may without loss of generality assume that no point in $X$ has a neighborhood of cardinality $\kappa$. We now may proceed to construct a downward space by induction.

Assume for $i<n$ we have discrete spaces $Y_{i} \subset X$ such that if $i<j<n$ then each point of $Y_{i}$ has a neighborhood whose intersection with $Y_{j}$ is empty, that each $Y_{i}$ has cardinality $\kappa_{i}$, and for each $i<n$ there is some $m_{i}$ such that each point of $Y_{i}$ has a neighborhood in $X$ of cardinality $\leqq \kappa_{m_{i}}$, say to the point $y$ we have assigned the small neighborhood $u_{y}$. Let $Z_{n}=\mathbf{U}_{i<n} \mathbf{U}_{y_{\in} Y_{i}} u_{y}$. Since $\left|Z_{n}\right|<\kappa$ there is some $m_{n}$ such that $\left\{x \in X-Z_{n}: x\right.$ has a neighborhood of cardinality $\left.\leqq \kappa_{m_{n}}\right\}=B_{n}$ has cardinality $\geqq \kappa_{n}$. But then for some $k_{n}, B_{n} \cap X_{k_{n}} \geqq \kappa_{n}$. Let $Y_{n} \subset B_{n} \cap X_{k_{n}}$ of the required cardinality, and to each $y \in Y_{n}$ associate a neighborhood $u_{y}$ of cardinality $\leqq \kappa_{m_{n}}$. By construction, the space $Y=\bigcup_{n \in \omega} Y_{n}$ is a downward subspace of $X$.

Theorem 10 is proved. But in the proof we actually learn more, since if $X$ has a subspace $X^{\prime}$ of cardinality $\kappa$ in which either Case 1 or 2 holds, spread is attained. Thus a counterexample must contain a space which is not only downward, but in which every subspace of cardinality $\kappa$ satisfies Case 3 of the proof of the theorem.

In fact a theorem of Juhasz and Hajnal's tells us more.

THEOREM 13. If $X$ is a strongly Hausdorff space whose spread has countable cofinality, $X$ attains its spread. (A strongly Hausdorff space is a Hausdorff space in which every infinite subset has an infinite subset which can be separated by a disjoint open family in the original space.)

The proof of Theorem 13 is given in Juhasz [6] and in its light the question of attaining a spread of countable cofinality reduces to the question: does every space whose spread has countable cofinality have a strongly Hausdorff subspace with the same spread? Actually, 
since every known example of a Hausdorff space which is not strongly Hausdorff is essentially countable (i.e. a countable space tacked on to any other space which is strongly Hausdorff) an open question of considerable interest is the following: for any cardinal $\kappa$ is there a Hausdorff space of cardinality $\kappa$ such that no subspace of cardinality $\kappa$ is strongly Hausdorff?

\section{REFERENCES}

1. W. B. Easton, Powers of regular cardinals, Ann. Math. Logic, 1 (1970), 139-178.

2. H. Hajnal and I. Juhasz, Discrete subspaces of topological spaces, Indag. Math., 29 (1967), 343-356.

3. - Discrete subspaces of topological spaces II, Indag. Math., 31 (1969), 18-30.

4. - Some remarks on a property of topological cardinal functions, Acta Math. Acad. Sci. Hung., 20 (1969), 25-37.

5 . - A consistency result concerning hereditarily $\alpha$-separable spaces, Proc. Bolyai Janos Math. Soc. Coll., Keszthely, 1972.

6. I. Juhasz, Cardinal Functions in Topology, Amsterdam, 1971

Received November 26, 1974 and in revised form February 5, 1975.

Wellesley College 



\section{PACIFIC JOURNAL OF MATHEMATICS}

\section{EDITORS}

RICHARD ARENS (Managing Editor)

University of California

Los Angeles, California 90024

\section{J. DugundJI}

Department of Mathematics University of Southern California Los Angeles, California 90007

D. Gilbarg and J. Milgram

Stanford University

Stanford, California 94305
University of Washington Seattle, Washington 98105

\section{ASSOCIATE EDITORS}
E. F. BECKENBACH
B. H. NeumanN
F. WolF
K. YoShIDA

\section{SUPPORTING INSTITUTIONS}

\author{
UNIVERSITY OF SOUTHERN CALIFORNIA \\ STANFORD UNIVERSITY \\ UNIVERSITY OF TOKYO \\ UNIVERSITY OF UTAH \\ WASHINGTON STATE UNIVERSITY \\ UNIVERSITY OF WASHINGTON \\ $\stackrel{*}{*} \stackrel{*}{*} \stackrel{*}{ }{ }^{*}$ AMERICAN MATHEMATICAL SOCIETY
}

The Supporting Institutions listed above contribute to the cost of publication of this Journal, but they are not owners or publishers and have no responsibility for its content or policies.

Mathematical papers intended for publication in the Pacific Journal of Mathematics should be in typed form or offset-reproduced, (not dittoed), double spaced with large margins. Underline Greek letters in red, German in green, and script in blue. The first paragraph or two must be capable of being used separately as a synopsis of the entire paper. Items of the bibliography should not be cited there unless absolutely necessary, in which case they must be identified by author and Journal, rather than by item number. Manuscripts, in triplicate, may be sent to any one of the editors. Please classify according to the scheme of Math. Reviews, Index to Vol. 39. All other communications should be addressed to the managing editor, or Elaine Barth, University of California, Los Angeles, California, 90024.

The Pacific Journal of Mathematics expects the author's institution to pay page charges, and reserves the right to delay publication for nonpayment of charges in case of financial emergency.

100 reprints are provided free for each article, only if page charges have been substantially paid. Additional copies may be obtained at cost in multiples of 50 .

The Pacific Journal of Mathematics is issued monthly as of January 1966. Regular subscription rate: $\$ 72.00$ a year (6 Vols., 12 issues). Special rate: $\$ 36.00$ a year to individual members of supporting institutions.

Subscriptions, orders for back numbers, and changes of address should be sent to Pacific Journal of Mathematics, 103 Highland Boulevard, Berkeley, California, 94708.

\section{PUBLISHED BY PACIFIC JOURNAL OF MATHEMATICS, A NON-PROFIT CORPORATION}

Printed at Kokusai Bunken Insatsusha (International Academic Printing Co., Ltd.), 270, 3-chome Totsuka-cho, Shinjuku-ku, Tokyo 160, Japan.

\section{Copyright (C) 1975 by Pacific Journal of Mathematics} Manufactured and first issued in Japan 


\section{Pacific Journal of Mathematics}

\section{Vol. 57, No. $2 \quad$ February, 1975}

Norman Larrabee Alling, On Cauchy's theorem for real algebraic curves with boundary .......

Daniel D. Anderson, A remark on the lattice of ideals of a Prüfer domain ..................

Dennis Neal Barr and Peter D. Miletta, A necessary and sufficient condition for uniqueness of

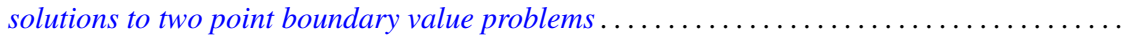

Ladislav Beran, On solvability of generalized orthomodular lattices . . . . . . . . . . ........

L. Carlitz, A three-term relation for some sums related to Dedekind sums . . . . . . . . . .....

Arthur Herbert Copeland, Jr. and Albert Oscar Shar, Images and pre-images of localization

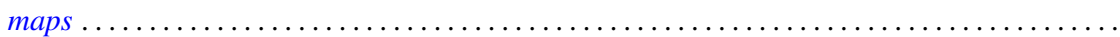

G. G. Dandapat, John L. Hunsucker and Carl Pomerance, Some new results on odd perfect

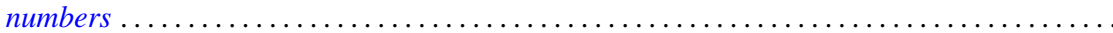

M. Edelstein and L. Keener, Characterizations of infinite-dimensional and nonreflexive

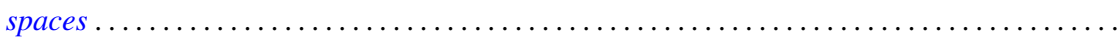

Francis James Flanigan, On Levi factors of derivation algebras and the radical embedding

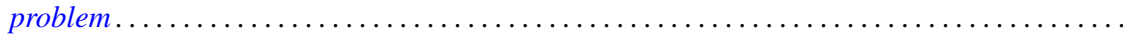

Harvey Friedman, Provable equality in primitive recursive arithmetic with and without

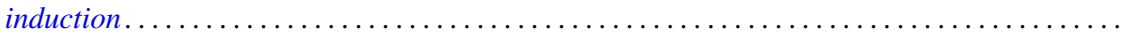

Joseph Braucher Fugate and Lee K. Mohler, The fixed point property for tree-like continua with

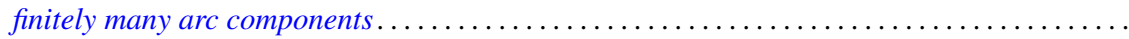

John Norman Ginsburg and Victor Harold Saks, Some applications of ultrafilters in

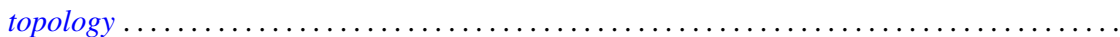

Arjun K. Gupta, Generalisation of a "square" functional equation .....................

Thomas Lee Hayden and Frank Jones Massey, Nonlinear holomorphic semigroups ..........

V. Kannan and Thekkedath Thrivikraman, Lattices of Hausdorff compactifications of a locally

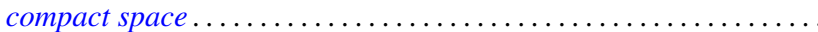

J. E. Kerlin and Wilfred Dennis Pepe, Norm decreasing homomorphisms between group

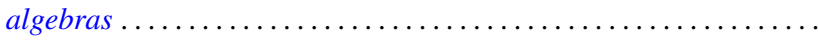

Young K. Kwon, Behavior of $\Phi$-bounded harmonic functions at the Wiener boundary ...

Richard Arthur Levaro, Projective quasi-coherent sheaves of modules .

Chung Lin, Rearranging Fourier transforms on groups...........................

David Lowell Lovelady, An asymptotic analysis of an odd order linear differential equation . . 4475

Jerry Malzan, On groups with a single involution .......................... 481

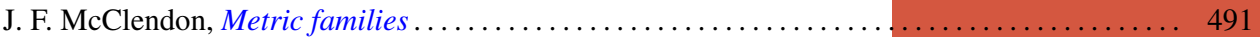

Carl Pomerance, On multiply perfect numbers with a special property .

Mohan S. Putcha and Adil Mohamed Yaqub, Polynomial constraints for finiteness of semisimple rings. .

Calvin R. Putnam, Hyponormal contractions and strong power convergence . . . . . . . . . 531

Douglas Conner Ravenel, Multiplicative operations in $\mathrm{BP} * \mathrm{BP} \ldots \ldots \ldots \ldots \ldots \ldots \ldots \ldots \ldots .539$

Judith Roitman, Attaining the spread at cardinals which are not strong limits . . . . . . . . . 545

Kazuyuki Saitô, Groups of *-automorphisms and invariant maps of von Neumann algebras . . . 553

Brian Kirkwood Schmidt, Homotopy invariance of contravariant functors acting on smooth

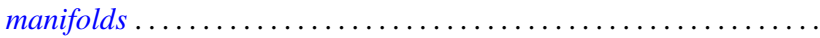

Kenneth Barry Stolarsky, The sum of the distances to $N$ points on a sphere.

Mark Lawrence Teply, Semiprime rings with the singular splitting property.

J. Pelham Thomas, Maximal connected Hausdorff spaces..............

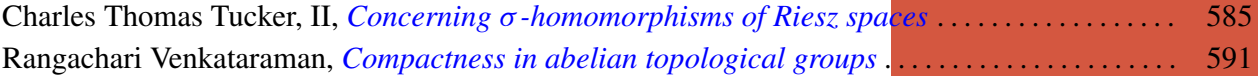

William Charles Waterhouse, Basically bounded functors and flat sheaves . . . . . . . . . . . 597

David Westreich, Bifurcation of operator equations with unbounded linearized part ......... 611

William Robin Zame, Extendibility, boundedness and sequential convergence in spaces of 